\title{
Disseminated Fusarium infection in a patient with acute lymphoblastic leukemia: A case report and review of the literature
}

\author{
YI-SHENG LIU $^{1,2}$, NING-CHI WANG ${ }^{3}$, REN-HUA YE ${ }^{1}$ and WEI-YAO KAO ${ }^{1,4}$ \\ ${ }^{1}$ Division of Hematology and Oncology, Department of Medicine, Tri-Service General Hospital, \\ National Defense Medical Center, Taipei 114; ${ }^{2}$ Department of Medicine, Taichung Armed Forces General Hospital, \\ Taichung 41152; ${ }^{3}$ Division of Infectious Disease, Department of Medicine, Tri-Service General Hospital, \\ National Defense Medical Center, Taipei 114; ${ }^{4}$ Division of Hematology and Oncology, Department of Medicine, \\ Taipei Tzu Chi General Hospital, Taipei 23142, Taiwan, R.O.C.
}

Received May 21, 2013; Accepted November 25, 2013

DOI: $10.3892 / 01.2013 .1738$

\begin{abstract}
Fusarium is a common soil mold. In severely immunocompromised patients, this fungus may cause disseminated disease and is often confused with Aspergillus, as the two pathogens have similar histopathological appearances. Disseminated Fusarium infection may cause significant morbidity and mortality in immunocompromised patients. The current case report presents a 20 -year-old male with acute lymphoblastic leukemia who developed disseminated Fusarium infection during induction chemotherapy. Early diagnosis and treatment is extremely important since the mortality rate is extremely high in such patients. The clinician must consider that the clinical presentation of Fusarium infection resembles that of Aspergillus. There is no optimal treatment for patients with Fusarium infection; however, combination antifungal therapy may have benefit without significant toxicity.
\end{abstract}

\section{Introduction}

Invasive fungal infections are leading causes of mortality and morbidity in patients with hematological malignancies and prolonged neutropenia following chemotherapy $(1,2)$. In general, fluconazole is used as a prophylactic antifungal treatment for patients with hematological malignancies who are predicted to exhibit prolonged neutropenia following aggressive chemotherapy. Thus, mold infections become more common in patients with fluconazole prophylaxis and Fusarium is the second most common cause of mold infection. The incidence of Fusarium spp. infections in patients with acute leukemia in

Correspondence to: $\mathrm{Dr}$ Wei-Yao Kao, Division of Hematology and Oncology, Department of Medicine, Tri-Service General Hospital, National Defense Medical Center, 325, Section 2, Cheng-Gung Road, Taipei 114, Taiwan, R.O.C

E-mail:wykao@tpts5.seed.net.tw

Key words: Fusarium, Aspergillus, acute leukemia, neutropenia
Europe is $0.06 \%$ (3) and was $1.2 \%$ among 750 allogeneic and $0.2 \%$ among 1,537 autologous marrow transplant recipients in the United States (4). Disseminated fusariosis accounts for $70 \%$ of Fusarium infections in immunocompromised patients, particularly in patients with acute leukemia with prolonged and profound neutropenia, and patients undergoing hematopoietic stem cell transplantation (5).

\section{Case report}

A 20-year-old male was diagnosed with acute lymphoblastic leukemia, precursor $\mathrm{B}$ cell type and received induction chemotherapy with TPOG-ALL-2002 VHR protocol (6) with partial remission in the induction course. The patient did not finish the consolidation course due to prolonged neutropenia. Later, the patient relapsed and received chemotherapy with FLAG-IDA (idarubicin, fludarabine, cytarabine and G-CSF) (7). The patient exhibited prolonged febrile neutropenia for more than one month during the initial course of chemotherapy, but exhibited rapid relapse again, soon following neutropenia recovery. In the second course of treatment, the patient received $400 \mathrm{mg}$ oral fluconazole daily as an antifungal prophylaxis. The febrile neutropenia was found two days later. Antibiotic treatment with imipenem/cilastatin (500 mg, i.v., every $6 \mathrm{~h}$ ), vancomycin $(1,000 \mathrm{mg}$, i.v., every $12 \mathrm{~h}$ ) and micafungin (100 mg, i.v., daily) was then administrated. After four days, multiple skin lesions, starting from the legs and spreading to the face and upper extremities were identified. The lesions exhibited necrotic centers surrounded by spreading erythema (Fig. 1). The lesions worsened and the antifungal treatment was replaced with caspofungin $(100 \mathrm{mg}$, i.v., daily). A biopsy of the skin lesions showed the presence of hyphae occupying the vascular space. The Gomori methanamine silver and periodic acid-Schiff stains were positive. The histopathological diagnosis was angioinvasive aspergillosis and treatment with voriconazole $(200 \mathrm{mg}$, i.v., every $12 \mathrm{~h}$ ) was initiated in addition to caspofungin with poor response. The amphotericin B was not administered due to ethical issues (the patient and family refused further aggressive treatment owing to refractory acute lymphoblastic leukemia with poor therapeutic response). A high-resolution 


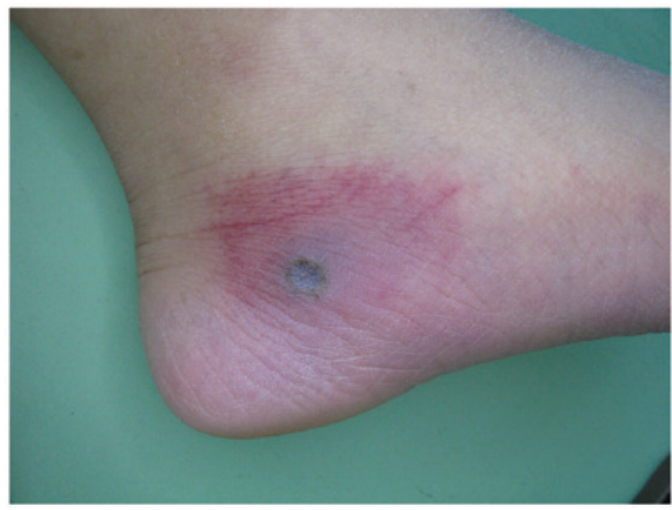

Figure 1. Classical single lesion with indurated erythematous papule and central necrosis.

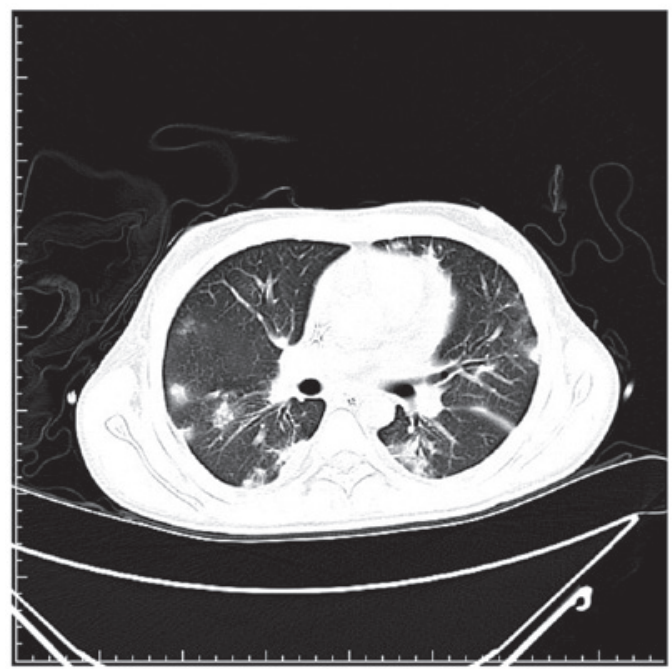

Figure 2. Radiological observations may indicate non-specific infiltrates to nodular and/or cavity formation.

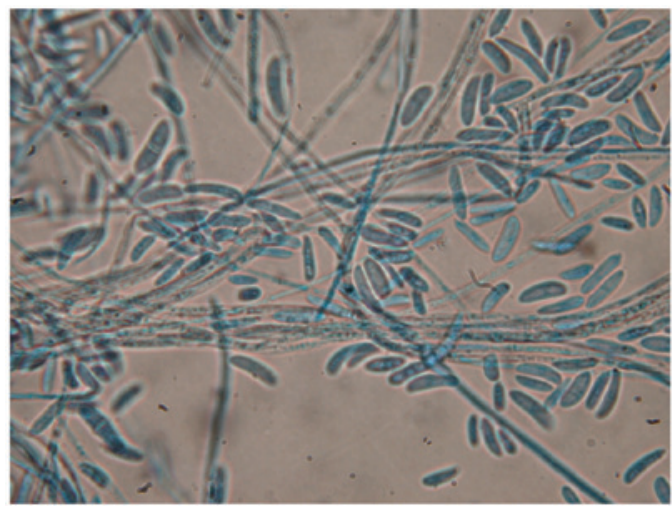

Figure 3. Microscopically, Fusarium filaments were hyaline, septate and 3to $8-\mu \mathrm{m}$ in diameter, typically branching at acute or right angles.

computed tomography scan of the lungs found multiple speculated and round consolidative densities in the lungs (Fig. 2). After three weeks, the sputum and skin tissue cultures finally demonstrated Fusarium spp. colonization (Fig. 3). The patient succumbed to the disease two months later. Written consent was provided from the patient's family.

\section{Discussion}

Fusarium is widely distributed in soil, plants and air and is common in tropical and temperate regions. This pathogen can cause a broad spectrum of human diseases, which may be locally invasive or disseminated. In immunocompromised patients, particularly in high-risk patients (those with prolonged and profound neutropenia), Fusarium infection is typically invasive and disseminated (5). In a previous study of invasive fusariosis in 84 patients with hematological malignancies, patients with acute leukemia occurred most frequently (56\%) and the majority of patients $(83 \%)$ were neutropenic at diagnosis (8). The incidence of Fusarium spp. infection in patients with acute leukemia in Europe is $0.06 \%$ (3). Previously, in a 10 -year follow-up study from a US institution, the incidence of Fusarium infection was $1.2 \%$ among 750 allogeneic and $0.2 \%$ among 1,537 autologous marrow transplant recipients (4).

Fusarium infection in immunocompromised patients is airborne or inoculated through the breakdown of the skin barrier. Skin lesions may involve any skin sites, with predominance in the extremities. Fusarium infection presents more frequently as metastatic skin lesions with initial presentation of subcutaneous lesions, to erythematous indurations, followed by target-like central necrotic lesions (4). The lesions are evoked rapidly within one to five days, at various stages of evolution and occasionally with myalgias (9). Skin biopsies are extremely easy to perform and confirm clinical suspicion. However, the histopathological images of skin lesions resemble those of Aspergillus infections. The two infections exhibit vascular invasion and branching septate hyphae (4); therefore, identification from Aspergillus may be difficult.

As with the case of Aspergillus, the radiological observations of pulmonary Fusarium infection are non-specific, including non-specific infiltrates to nodular and/or cavity formation, depending on the timing of the examination (9). The prognosis is considerably worse in patient pulmonary infiltrates (10).

The definite diagnosis of Fusarium infection requires Fusarium spp. isolation from blood and tissue culture (such as the skin, lungs and sinuses). However, the Fusarium species may contaminate laboratory specimens and pseudo-outbreaks of fusariosis may occur (11). Thus, the clinician must be cautious of interpretation. Unlike Aspergillus, the Fusarium species may be isolated from cultures of blood samples in $40 \%$ of cases. With the presence of disseminated skin lesions, the presence of the fungus in blood cultures may increase to $56 \%$ (8). In the majority of cases, fungemia is likely to develop in a median of five days (range, one to 10 days) following the appearance of skin lesions (9). However, in the present case, the blood culture did not yield Fusarium species despite disseminated skin lesions.

For patients with persistently febrile neutropenia, empiric treatment with caspofungin is initially recommended and liposomal amphotericin B is an alternative choice (12). There is no treatment guideline for Fusarium infection. Nucci et al (8) suggested high-dose amphotericin B or lipid formation of amphotericin B since specific Fusarium species may be resistant to azoles. The greatest challenge to clinicians is the early diagnosis of the Fusarium infection and preemptive treatment, since the histopathology of Fusarium may be confused with 
Aspergillus and the galactomannan test may not be useful for distinguishing Fusarium from Aspergillus (13). Despite aggressive treatment, the survival rate of the Fusarium infection in patients with persistent neutropenia is only $4 \%$ (8). Early granulocyte recovery is significantly associated with favorable response and survival and growth factors (G-CSF or GM-CSF) or granulocyte transfusions, which may shorten the neutropenic period to aid immunity recovery (15). Combination antifungal therapy is well-tolerated with acceptable minor toxicity $(14,15)$ and, theoretically, may have benefits to stabilizing the infection and preventing fatal progression. However, such data are limited in hematological patients with Fusarium infection. In a previous report of immunocompromised patients with disseminated Fusarium infection, 70\% of patients (14 cases) exhibited a positive response to combination antifungal therapy (16). In one multicenter retrospective study of 61 hematological patients with confirmed or predicted invasive mold infections, comparing liposomal amphotericin B plus caspofungin, liposomal amphotericin B plus a triazole and voriconazole plus one echinocandin drug, no statistical differences were identified among these groups at the end of treatment and 12-week survival (15). In the current case, the patient was recommended amphotericin B plus caspofungin or amphotericin B plus voriconazole treatment in combination; however, the patient and family refused due to ethical issues. The optimal treatment for patients with Fusarium infection requires further investigation and well-designed clinical trails to address this issue.

\section{References}

1. Pagano L, Caira M, Candoni A, et al: The epidemiology of fungal infections in patients with hematologic malignancies: the SEIFEM-2004 study. Haematologica 91: 1068-1075, 2006.

2. Böhme A, Ruhnke M, Buchheidt D, et al: Treatment of invasive fungal infections in cancer patients - recommendations of the Infectious Diseases Working Party (AGIHO) of the German Society of Hematology and Oncology (DGHO). Ann Hematol 88: 97-110, 2009.
3. Girmenia C, Pagano L, Corvatta L, et al: The epidemiology of fusariosis in patients with haematological diseases. Gimema Infection Programme. Br J Haematol 111: 272-276, 2000.

4. Boutati EI and Anaissie EJ: Fusarium, a significant emerging pathogen in patients with hematologic malignancy: ten years experience at a cancer center and implications for management. Blood 90: 999-1008, 1997.

5. Nucci M and Anaissie E: Fusarium infections in immunocompromised patients. Clin Microbiol Rev 20: 695-704, 2007.

6. Liang DC, Yang CP, Lin DT, et al: Long-term results of Taiwan Pediatric Oncology Group studies 1997 and 2002 for childhood acute lymphoblastic leukemia. Leukemia 24: 397-405, 2010.

7. Fleischhack G, Hasan C, Graf N, Mann G and Bode U: IDA-FLAG (idarubicin, fludarabine, cytarabine, G-CSF), an effective remission-induction therapy for poor-prognosis AML of childhood prior to allogeneic or autologous bone marrow transplantation: experiences of a phase II trial. Br J Haematol 102: 647-655, 1998.

8. Nucci M, Anaissie EJ, Queiroz-Telles F, et al: Outcome predictors of 84 patients with hematologic malignancies and Fusarium infection. Cancer 98: 315-319, 2003.

9. Dignani MC and Anaissie EJ: Human fusariosis. Clin Microbiol Infect 10 (Suppl 1): 67-75, 2004.

10. Chaoui D, Legrand O, Roche N, et al: Incidence and prognostic value of respiratory events in acute leukemia. Leukemia 18: 670-675, 2004.

11. Grigis A, Farina C, Symoens F, Nolard N and Goglio A: Nosocomial pseudo-outbreak of Fusarium verticillioides associated with sterile plastic containers. Infect Control Hosp Epidemiol 21: 50-52, 2000.

12. Rüping MJ, Vehreschild JJ and Cornely OA: Antifungal treatment strategies in high risk patients. Mycoses 51 (Suppl 2): 46-51, 2008

13. Tortorano AM, Esposto MC, Prigitano A, Grancini A, Ossi C, Cavanna C and Cascio GL: Cross-reactivity of Fusarium spp. in the Aspergillus Galactomannan enzyme-linked immunosorbent assay. J Clin Microbiol 50: 1051-1053, 2012.

14. Cetkovsky P, Kouba M, Markova M, et al: Combination of voriconazole and anidulafungin as primary therapy in hematologic patients. Biol Blood Marrow Transplant 16: S264, 2010.

15. Rojas R, Molina JR, Jarque I, et al: Outcome of antifungal combination therapy for invasive mold infections in hematological patients is independent for chosen combination. Mediterr J Hematol Infect Dis 4: e2012011, 2012.

16. Liu JY, Chen WT, Ko BS, et al: Combination antifungal therapy for disseminated fusariosis in immunocompromised patients: a case report and literature review. Med Mycol 49: 872-878, 2011. 